\title{
DIFFERENTIAL SEARCH ALGORITHM BASED EDGE DETECTION
}

\author{
M. A. Gunen ${ }^{\text {b, }}{ }^{*}$, P. Civicioglu ${ }^{\text {a }}$, E. Beşdok ${ }^{\text {b }}$ \\ ${ }^{\text {a }}$ Erciyes University, Faculty of Aeronautics and Astronautics, Department of Aircraft Electrics and Electronics, \\ 38039 Kayseri, Turkey, civici@erciyes.edu.tr \\ ${ }^{\mathrm{b}}$ Erciyes University, Engineering Faculty, Department of Geomatics Engineering, \\ 38039 Kayseri, Turkey, (akif,ebesdok)@erciyes.edu.tr
}

Commission VII, WG VII/6

KEY WORDS: Differential Search Algorithm, Edge Detection, Image Fusion.

\begin{abstract}
:
In this paper, a new method has been presented for the extraction of edge information by using Differential Search Optimization Algorithm. The proposed method is based on using a new heuristic image thresholding method for edge detection. The success of the proposed method has been examined on fusion of two remote sensed images. The applicability of the proposed method on edge detection and image fusion problems have been analysed in detail and the empirical results exposed that the proposed method is useful for solving the mentioned problems.
\end{abstract}

\section{INTRODUCTION}

Edge detection is one of the mostly used image segmentation operation in image processing applications (Çivicioğlu, Alçı, 2004), (Russo, 1998), (Besdok, et.all., 2004 a,b,c), High frequency impulsive noises (Civicioglu, et.all. 2004 a,b), (Civicioglu, 2009), additive noises or intensity changes point out existence of edge information. In classical edge detection operations, a linear edge detection convolution kernel and image are convolved in order to approximate image gradients. In general, edge detection kernels are used to simulate first or second derivative of the concerned image. By this way intensity discontinuities can be detected easily by using a predefined threshold (Kurban, et.all., 2014).

Edges of images are marked with intensity discontinuities or rapid variations in intensity values. In general, edge detection processes must fulfil some simple requirements, such as; 1 . Edges must be detected at correct locations, 2. False edge detection rate has to be too low. Edge detection problem has been intensively investigated in recent years. Hence, there are lots of efficient techniques developed for edge detection, such as gradient based methods and thresholding/clustering based methods, which are applied at edge extraction, image-separation and classification.

Fuzzy C-Means and SOM neural networks based clustering methods have been used in many of the edge detection problems (Bezdek, et.all., 1984) (Mingoti, et.all., 2006). Artificial Intelligence tools are relatively flexible, quite robust but they need much more runtime contrary to classical methods in real time applications. They are also too computationalextensive methods. Therefore, gradient or clustering based methods were preferred in the literature for the edge extraction from images. One of the first version of [3×3] sized edge detection convolution kernels were introduced by Frei and Chen (Çivicioğlu, Alçı, 2004c). The most frequently used image gradient simulators for edge detection are gradient-based methods, such as Sobel, Prewitt, and Roberts (Kanopoulos, et.all, 1988), (Cherri, Karim, 1989), (Hsieh, et.all., 1997).
There are several methods proposed in the literature for detecting suboptimum threshold value for edge detection from image gradient information (Kurban, et.all., 2014).

The gradient of image $I=f(u, v)$ is computed by using Eq.1:

$\nabla I=\left[\begin{array}{lll}\frac{\partial I}{\partial u} & , & \frac{\partial I}{\partial v}\end{array}\right]$

The magnitude of image gradient is computed by using Eq.2:

$M=\sqrt{\left(\frac{\partial I}{\partial u}\right)^{2}+\left(\frac{\partial I}{\partial v}\right)^{2}}$

The direction information of gradient is computed by using Eq.3:

$t=\tan ^{-1}\left(\frac{\partial I}{\partial v} \times\left(\frac{\partial I}{\partial u}\right)^{-1}\right)$

One of the orthogonal directions can be used in order to obtain image gradients, but the mostly used directions are $u$ and $v$ cartesian directions. The basic convolution kernel of Roberts edge detector is given in Eq. 4. Similarly, the basic convolution kernel of Sobel and Prewitt edge detectors are given in Eq.5 and Eq.6, respectively.

$$
\begin{aligned}
& k_{\text {roberts }}=\left[\begin{array}{cc}
0 & 1 \\
-1 & 0
\end{array}\right] \\
& k_{\text {prewitt }}=\left[\begin{array}{lll}
-1 & 0 & 1 \\
-1 & 0 & 1 \\
-1 & 0 & 1
\end{array}\right]
\end{aligned}
$$


$k_{\text {sobel }}=\left[\begin{array}{lll}-1 & 0 & 1 \\ -2 & 0 & 2 \\ -1 & 0 & 1\end{array}\right]$

In this paper, a new edge detection algorithm has been proposed where the detected edges have been used to improve image registration of two test images (Hsieh, et.all., 1997). The images were acquired from the optic bands of Tubitak-Rasat and Landsat-8 satellites (Erdogan, et.all., 2016), (Roy, et.all., 2014). Images were projected same topographic surface by using SRTM database.

The rest of the paper is organized as follows: The proposed method is given in Section 2. Experiments and Conclusions are presented in Section 3 and Section 4, respectively.

\section{PROPOSED EDGE DETECTOR}

Let $u=\left\{\begin{array}{lll}x_{1:(c-1)} & x_{c} & x_{(c+1): n}\end{array}\right\}$ denote $n \times n$ sized image segment, where $n$ is an odd-number. Here, $x_{c}$ denotes center pixel of the $n \times n$ sized sliding-window. Let $v=u \backslash\left\{x_{c}\right\}$ and $T_{2}=\left|\left\{\left|v-x_{c}\right| \geq T_{1}\right\}\right|$ as in [1-10]. The $T_{2}$ pseudo-image of Rband of Rasat test image (see Fig.1) for $T_{1}=20$ and $n=3$ is illustrated at Fig. 2. The result of $T_{2}$ range-filter for the related test image shows extreme valued local intensity changes, which most probably belong to edge-pixels.

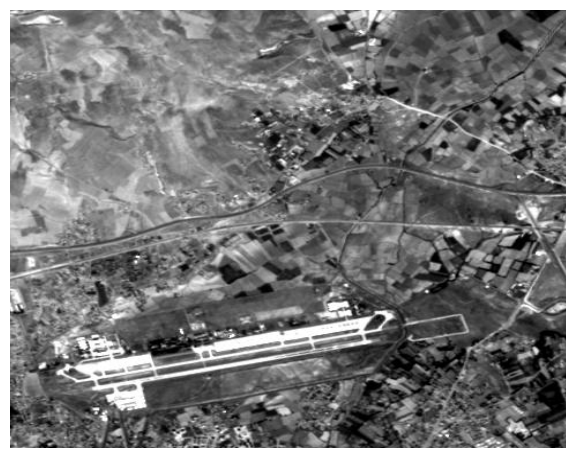

Figure 1: Red band of Rasat test image.

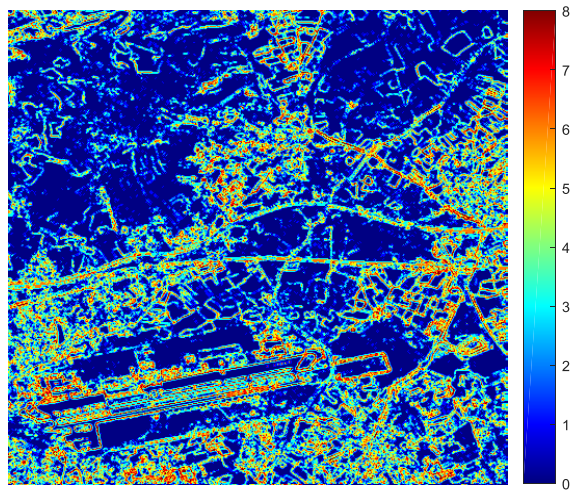

Figure 2: The pseudo-image of R-band of Rasat test image for $T_{1}=20$ and $n=3$.

The result of $T_{2}$ range-filter is clustered into two classes by using Eq.7 as:
$\underset{S}{\arg \min } \sum_{i=1}^{k} \sum_{x \in S_{i}}\left\|x-\mu_{i}\right\|$

where $\mu_{i=1: 2}$ centers were obtained by using bijective Differential Search Algorithm (DSA) (Civicioglu, 2012, 2013a, 2013b).

DSA is a new evolutionary optimization algorithm which has been proposed for minimizing real-valued numerical optimization problems. General structure of DSA is given in Fig. 3.

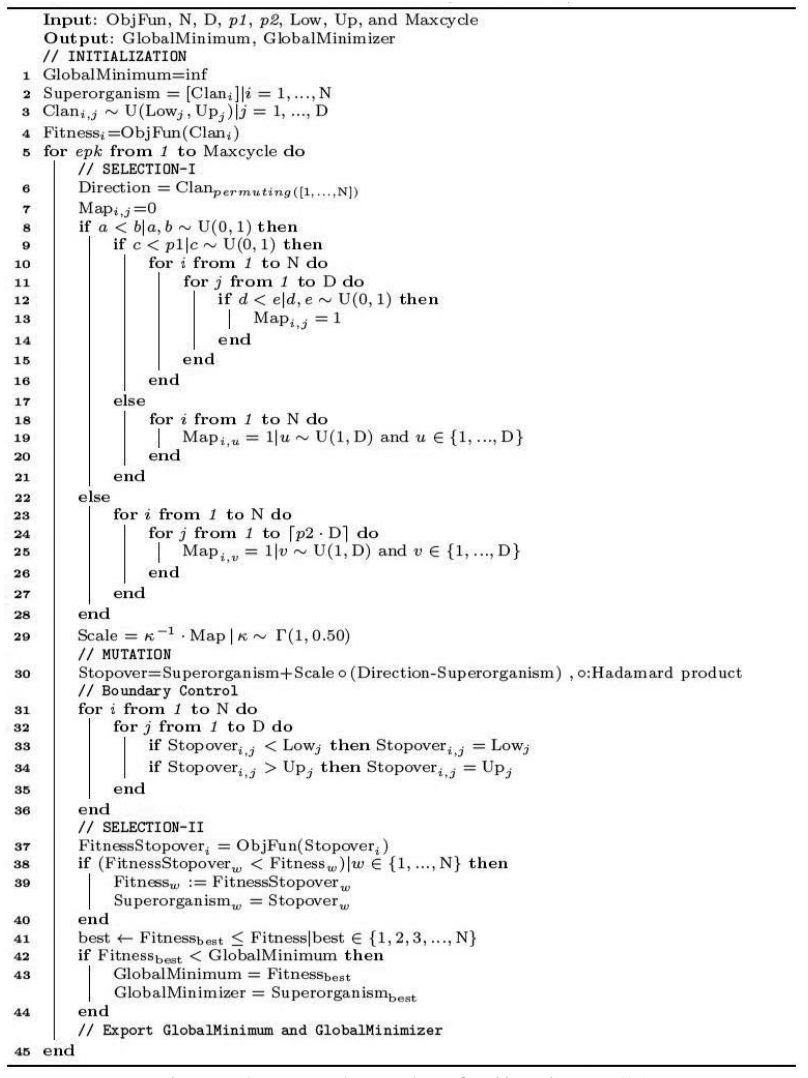

Figure 3: Pseudo code of Bijective DSA.

Due to its improved global search ability, DSA gives more suboptimized clustering results than k-means, fuzzy c-means and isodata methods for image clustering problems.

The clustered image, which also corresponds to binary image of the concerned image, is illustrated in Fig. 4.

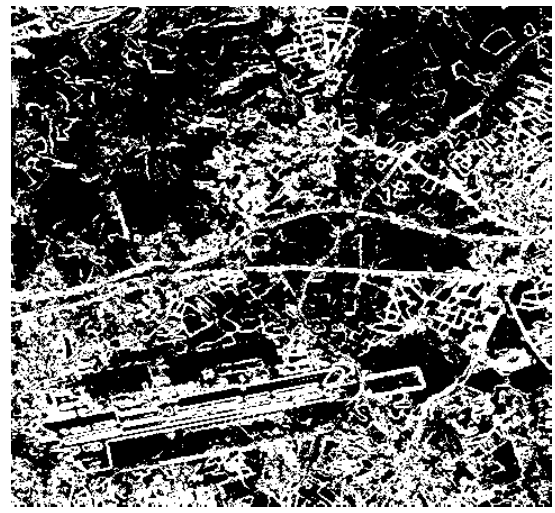

Figure 4: Binary version of R-band of Rasat test image. 
After the computation of the binary image, its absolute-valued gradient magnitude components have been obtained. Then a morphological thinning operator has been applied to each binary gradient magnitude component in order to obtain thinner edges. Finally obtained thin edged images have been combined by using logic 'or' operator. The edge images of Sobel, Prewitt and the proposed method have been given in Fig. 5.

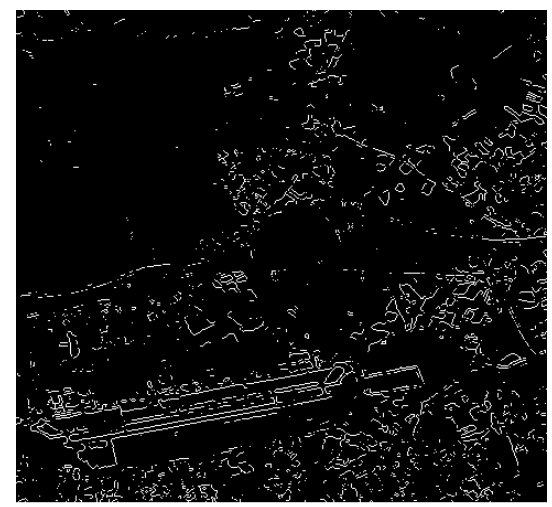

(a)

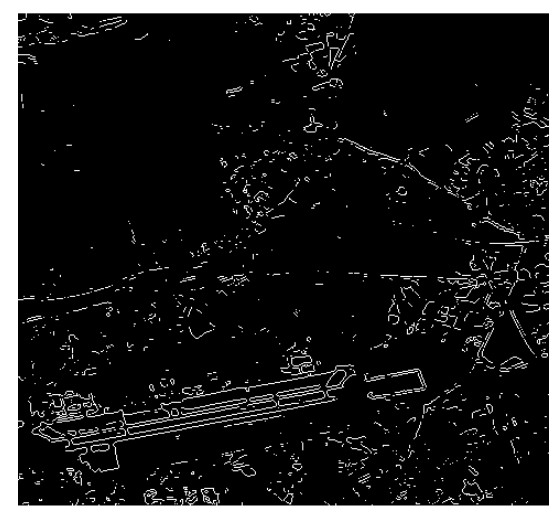

(b)

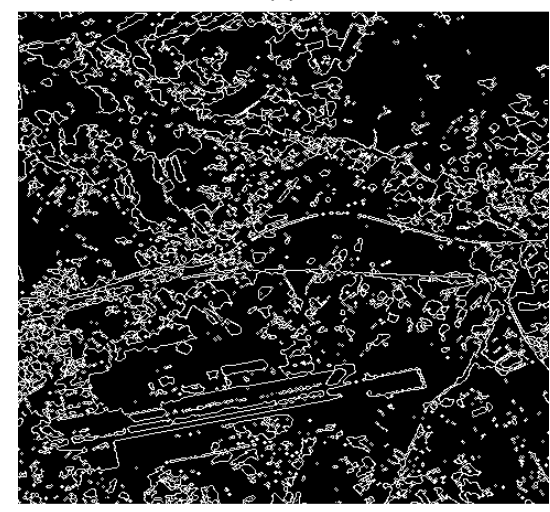

(c)

Figure 5: Edges of R-band of Rasat test image: (a) Sobel, (b) Prewitt, (c) Proposed Method.

Similarly, edge data of R-band of the second test image (i.e., Landsat- 8 image, see Fig. 6 (b)) have been computed by performing the procedure used for obtaining the edge pixels of R-band of the Rasat test image.

\section{WAVELETS BASED IMAGE FUSION}

Edge information conveyor pixels of the concerned images have been replaced with 255 at each band. After this process, the SURF features have been computed in order to define

homograph between images. An affine transformation model has been used to obtain 2D homography between the concerned images (Zhang, et.all., 2016), (Veelaert, Teelen, 2006). SURF features have been used to solve parameters of affine transformation by using a DSA based RANSAC method. The used homography detection scheme is given in Fig. 7. After obtaining of affine transformation parameters, Rasat test image has been correctly aligned to Landsat test image.

Image image registration problem is solved by using also least squares and numerical quality of achieved transformation is MSE $=0.015194$. The transformation quality of DSA based homography detection RANSAC [1-10] method has been obtained as $\mathrm{MSE}=0.015187$. In the experiments about the homography solving problem, we have used popsize $=10, \mathrm{dim}=6$ and epochs $=20000$ for DSA. After the homography solving phase, images have been registered. Then, the classical 2layered and 4-layered Daubechies Wavelets (Li, et.all., 1995, Daubechies, 1992.) have been used for the fusion of the concerned images. The image fusion results were given in Fig. 8 .

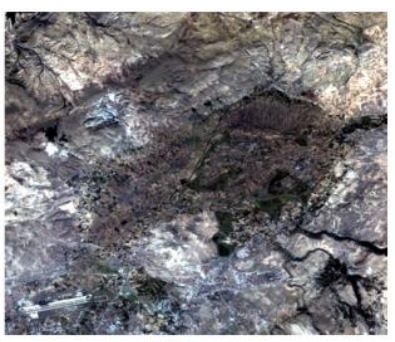

(a)

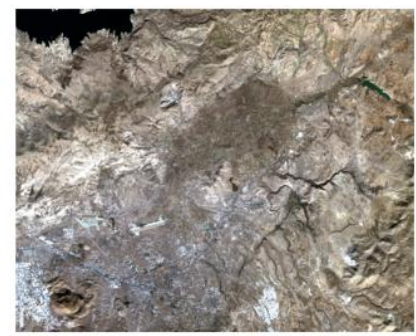

(b)

Figure 6: (a) Rasat RGB Image, (b) Landsat-8 RGB Image.

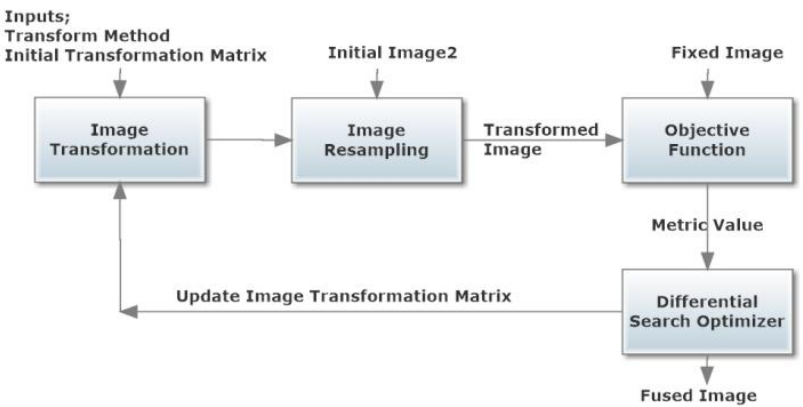

Figure 7: Proposed Method for image registration.

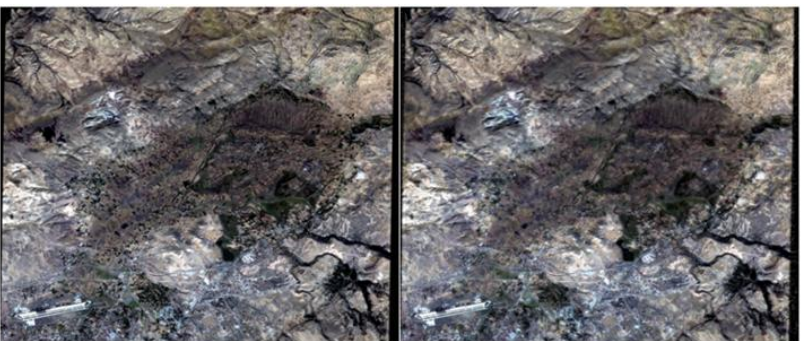

(a)

(b)

Figure 8: Daubechies Wavelets based fusion images; (a) 2layered wavelets, (b) 4-layered wavelets. 


\section{CONCLUSIONS}

In this paper, a new edge extraction method based on local intensity changes with DSA based clustering, has been presented. The success of the proposed method has been examined on fusion of two remote sensed images, Rasat and Landsat- 8 images. The obtained edges have been used to increase the detection rate of SURF features. This process has increased the accuracy of the detected affine holography parameters. As the image registration quality increased, false edge rates of image fusion have decreased for wavelets based image fusion.

\section{REFERENCES}

Besdok, E., Civicioglu, P., Alci, M., 2004. Impulsive noise suppression from highly corrupted images by using resilient neural networks. LNAI, 3070, pp.670-675.

Bezdek, J.C., Ehrlich, R., Full, W., 1984. FCM - the fuzzy cmeans clustering-algorithm. Computers \& Geosciences, 10 (23), pp. 191-203.

Civicioglu, P., 2009. Removal of random-valued impulsive noise from corrupted images. IEEE Transactions on Consumer Electronics, 55 (4), pp. 2097-2104.

Civicioglu, P., 2012. Transforming geocentric cartesian coordinates to geodetic coordinates by using differential search algorithm. Computers \& Geosciences, 46, pp. 229-247 Published: Sep 2012

Civicioglu, P., 2013a. Backtracking search optimization algorithm for numerical optimization problems. Applied Mathematics And Computation, 219 (15), pp. 8121-8144.

Civicioglu, P., Alci, M., Besdok, E., 2004a. Impulsive noise suppression from images with the noise exclusive filter. Eurasip Journal On Applied Signal Processing, 2004 (16), pp. 24342440.

Civicioglu, P., Alci, M., Besdok, E., 2004b. Using an exact radial basis function artificial neural network for impulsive noise suppression from highly distorted image databases. LNCS, 3261, pp. 383-391.

Civicioglu, P., Besdok, E., 2013b. A conceptual comparison of the cuckoo-search, particle swarm optimization, Differential Evolution and Artificial Bee Colony Algorithms. Artificial Intelligence Review, 39 (4), pp. 315-346.

Çivicioğlu B.P., Alçı, M., 2004c. Edge detection of highly distorted images suffering from impulsive noise. $A E U$ International Journal of Electronics and Communications, 58 (6), pp.413-419.

Daubechies, I., 1992. Ten Lectures on Wavelets, CBMS-NSF Conference Series in Applied Mathematics. Siam Ed.

Erdogan, M., Yilmaz, A., Eker, O., 2016. The georeferencing of rasat satellite imagery and some practical approaches to increase the georeferencing accuracy. Geocarto International, 31(6), pp.647-660.

Hsieh, J.W., Liao, H.Y.M., Fan, K.C., Et Al., 1997. Image registration using a new edge-based approach. Computer Vision and Image Understanding, 67 (2), pp. 112-130.

Kurban, T., Civicioglu, P., Kurban, R., Et Al., 2014. Comparison of Evolutionary And Swarm Based Computational
Techniques for Multilevel Color Image Thresholding, Applied Soft Computing, 23, pp. 128-143.

Li, H., Manjunath, B.S., Mitra, S.K., 1995. Multisensor image fusion using the wavelet transform. Graphical Models and Image Processing, 57 (3), pp. 235-245.

Mingoti, Sueli A.; Lima, Joab O. 2006. Comparing som neural network with fuzzy c-means, k-means and traditional hierarchical clustering algorithms. European Journal of Operational Research, 174 (3), pp. 1742-1759.

Roy, D.P., Wulder, M.A., Loveland, T.R., Et Al., 2014. Landsat-8: science and product vision for terrestrial global change research. Remote Sensing Of Environment, 145, pp. 154-172.

Russo, F., 1998. Edge detection in noisy images using fuzzy reasoning. IEEE Instrumentation and Measurement Technology Conference, Minnesota, USA, pp. 369-372.

Veelaert, P., Teelen, K., 2006. Consensus sets for affine transformation uncertainty polytopes. Computers \& Graphics, 30 (1), pp.77-85.

Zhang, Y., Zhou, L., Shang, Y., Zhang, X., Yu, Q., 2016. Contour model based homography estimation of texture-less planar objects in uncalibrated images. Pattern Recognition, 52, pp.375-383. 